\title{
System analysis of teratozoospermia mRNA profile based on integrated bioinformatics tools
}

\author{
TIANCHENG ZHANG ${ }^{1}$, JUN WU ${ }^{1}$, CAIHUA LIAO $^{2}$, ZHONG NI $^{3}$, JUFEN ZHENG $^{1,4}$ and FUDONG YU ${ }^{1}$ \\ ${ }^{1}$ Key Lab of Reproduction Regulation of NPFPC, Shanghai Institute of Planned Parenthood Research, \\ Institute of Reproduction and Development, Fudan University, Shanghai 300000; ${ }^{2}$ College of Biotechnology, \\ Guilin Medical University, Guilin, Guangxi Zhuang Autonomous Region 541004; ${ }^{3}$ Institute of Life Sciences, \\ Jiangsu University, Zhenjiang, Jiangsu 212013; ${ }^{4}$ Shanghai Key Laboratory of Reproductive Medicine, \\ Shanghai Jiao Tong University, Shanghai 200025, P.R. China
}

Received October 27, 2017; Accepted March 20, 2018

DOI: $10.3892 / \mathrm{mmr} .2018 .9112$

\begin{abstract}
RNA has an important role in spermatogenesis and the maintenance of fertility, and may act as a potential biomarker for the clinical diagnosis of infertility. In the present study, potential biomarkers associated with teratozoospermia were screened through systemic bioinformatics analysis. Initially, genome-wide expression profiles were downloaded from the Gene Expression Omnibus and primary analysis was conducted using $\mathrm{R}$ software, which included preprocessing of raw microarray data, transformation between probe ID and gene symbol and identification of differentially expressed genes. Subsequently, a functional enrichment analysis was conducted using the Database for Annotation, Visualization and Integrated Discovery to investigate the biological processes involved in the development of teratozoospermia. Finally, a protein-protein interaction network of notable differentially expressed genes was constructed and cross-analysis performed for multiple datasets, to obtain a potential biomarker for teratozoospermia. It was observed that $\mathrm{G}$ protein subunit $\beta 3$, $\mathrm{G}$ protein subunit $\alpha \mathrm{o} 1$ and $\mathrm{G}$ protein subunit $\mathrm{g}$ transducin 1 were upregulated and enriched using Kyoto Encyclopedia of Genes and Genomes (KEGG) in the network and in cross analysis. Furthermore, ribosomal protein S3 (RPS3), RPS5, RPS6, RPS16 and RPS23 were downregulated and enriched using KEGG in teratozoospermia. In conclusion, the results of the present study identified several mRNAs involved in sperm morphological development, which may aid in the understanding and treatment of infertility.
\end{abstract}

Correspondence to: Professor Jufen Zheng or Dr Fudong Yu, Key Lab of Reproduction Regulation of NPFPC, Shanghai Institute of Planned Parenthood Research, Institute of Reproduction and Development, Fudan University, 2140 Xietu Road, Shanghai 300000, P.R. China

E-mail: jf_zheng@sina.cn; ztctianna@163.com

E-mail: fdyush@163.com

Key words: teratozoospermia, mRNA profile, $\mathrm{G}$ protein, ribosomal protein

\section{Introduction}

Increasing attention has been focused on the function and significance of mRNA in sperm in light of the role it serves in sperm development and maintenance $(1,2)$. Thus, mRNAs that aid in detection of sperm abnormalities are potential biomarkers to evaluate the quality of sperm in the diagnosis and treatment of male infertility (3-5).

Teratozoospermia is a condition characterized by a large number of spermatozoa with abnormal morphology and is considered to be a factor that may result in male infertility (6). There are two manifestations of teratozoospermia: Monomorphic morphological defects and flagellum morphological defects $(6,7)$. Previous studies have demonstrated that abnormal expression of mRNA is a primary cause of abnormal sperm morphology (6-9). Kang-Decker et al (8) reported that male mice with ArfGAP with FG repeats 1 depletion were infertile due to a lack of acrosome formation. Casein kinase $2 \alpha 2$ knock-out male mice were also infertile due to abnormal morphology of the spermatid nucleus (9). Sptrx-2, expressed exclusively in human testis, was reported to be associated with flagellar anomalies (10). Allegrucci et al (11) also reported specific epigenetic signatures of flagellar anomalies. However, the specific mechanism underlying male infertility remains to be elucidated, as it is a complex process involving a large number of genes (12).

The rapid development of high-throughput technologies, including microarrays and RNA-sequencing has resulted in successful profiling of RNA expression, which enhances understanding of various diseases and helps further exploration of their underlying molecular mechanisms (13). HM et al (14) detected the gene expression of crossbred cattle sperm by microarray assessment and identified 305 genes that were significantly and differentially expressed. Hu et al (15) profiled long non-coding (lnc)RNA expression in male mice germ cells and revealed that a variety of lncRNAs may regulate male reproduction by serving as competing-endogenous RNAs to modulate the function of germ cells.

Exome sequencing analysis of two brothers with azoospermia demonstrated that the deficiency of homozygous 
serine peptidase inhibitor, Kazal type 2 is a factor in the development of azoospermia (16). However, to the best of our knowledge, there are limited studies that have systematically analyzed the gene expression profiles in patients with teratozoospermia using integration bioinformatics analysis. The present study obtained a gene expression dataset for teratozoospermia from the Gene Expression Omnibus (4) and performed systemic bioinformatics analysis, including identification of differentially expression genes (DEGs), functional enrichment analysis, co-expression network analysis and identified several significantly and differentially expressed biomarkers for teratozoospermia. The results of the present study may be beneficial in understanding the mechanism underlying teratozoospermia.

\section{Materials and methods}

Microarray data. The microarray dataset GSE6872 was downloaded from the GEO website (ncbi.nlm.nih.gov/gds/) which was based on the GPL570 platform. This dataset was submitted by Platts et al (17) and included 13 semen samples, collected from healthy fertile males. A total of 8 semen samples were collected from infertile patients with teratozoospermia without any other abnormal semen parameters.

Data preprocessing. The present study imported original CEL data into R (version 3.2.4, https://www.r-project.org/) and used an Affy R-package (Bioconductor version 3.6) to correct data background and data normalization. The mas5calls method for AffyBatch returns an ExpressionSet by multi probes which correspond to specific genes.

Differentially expressed gene selection. DEGs were identified between 13 healthy semen and 8 infertile semen samples, using the limma package (version 3.6, http://bioinf. wehi.edu.au/limma). False discovery rate (FDR)-value $<0.01$ and $\log 2$ fold changel $>2$ were selected as the cutoff values.

Functional annotation and pathway analysis of DEGs. The Database for Annotation, Visualization and Integrated Discovery (DAVID V6.8; https://david.ncifcrf.gov/) (18) was used to annotate and conclude gene lists or protein identifiers via comprehensive categorical data for Gene Ontology (GO) (19). In order to extensively evaluate connected pathways and biological processes associated with teratozoospermia, pathway enrichment analyses of DEGs were performed with the DAVID analysis system, with a threshold of $\mathrm{P} \leq 0.05$.

Protein interaction network and module analyses. The STRING database (http://string-db.org) was used to construct a protein-protein interaction (19) network of upregulated and downregulated DEGs, with a cutoff score of $>0.4$. The significant modules from the constructed PPI network of downregulated DEGs were selected using the ClusterONE plugin of the Cytoscape software v3.6.1 (cytoscape.org/plugins.html) with $\mathrm{P}<0.01$ considered to indicate a statistical significance.

\section{Results}

Analysis of DEGs. The expression profile data were pre-processed and then analyzed with the Affy package in $\mathrm{R}$ language. The whole gene expression was screened. Box plots following data standardization are presented in Fig. 1A and B. Median values in Fig. 1 are similar, which suggests a good degree of standardization. All RNA expression levels are presented in Fig. 2A. Hierarchical cluster analysis indicated that the 8 samples from patients with teratozoospermia and the 13 normal samples exhibited differing distributions. The results revealed that grouping was reasonable, and the data successfully underwent further analysis. Microarray data from the normal semen samples were compared with those from the teratozoospermia semen samples and a total of 2,392 DEGs were identified. There were 450 upregulated genes and 1,942 downregulated genes (Fig. 2B). The top 10 upregulated genes were heparan sulfate-glucosamine 3-sulfotransferase 3A1 (HS3ST3A1), XK related 4, armadillo-like helical domain containing 4, hydatidiform mole associated and imprinted (non-protein coding), WNT inhibitory factor 1, SLIT-ROBO Rho GTPase activating protein 2C, SLIT-ROBO Rho GTPase activating protein 2, DQ592442 (GenBank, https://www.ncbi. nlm.nih.gov/genbank/), monoacylglycerol O-acyltransferase 1 and LOC101928622. The most unregulated gene HS3ST3A1 is a component in heparan sulfate generation pathway that few studies reported to be associated with spermatogenesis $(20,21)$. The top 10 downregulated genes were zona pellucida binding protein (ZPBP), pancreatic progenitor cell differentiation and proliferation factor, microseminoprotein $\beta$, TSSK6 activating cochaperone, prolactin induced protein, transcription elongation factor A like 4, ribosomal protein (16) S5, ribosomal protein L7a pseudogene 12, semenogelin 1 and semenogelin 2 (Table I). The protein produced from the most downregulated gene, ZPBP, is usually located in the acrosome of spermatozoa (22). Abnormal morphogenesis is a major performance if patient lace of ZPBP expression (22).

Functional and pathway enrichment analysis. A total of 450 upregulated and 1,942 downregulated genes were uploaded to DAVID and GO analysis was conducted, with $\mathrm{P} \leq 0.05$ used to determine statistical significance. The top 10 GO terms enriched by up and downregulated genes are presented in Table II. The upregulated genes were primarily enriched in 'nervous system development', 'developmental processes', 'anatomical structural development', 'synapse', 'regulation of developmental processes', 'regulation of multicellular organismal development', 'synaptic membranes', 'positive regulation of developmental processes', 'regulation of multicellular organismal process' and 'postsynaptic membranes'. The top downregulated genes were primarily associated with 'protein targeting to the endoplasmic reticulum', 'membrane-enclosed lumen', 'nuclear part', 'SRP-dependent co-translational protein targeting to the membrane', 'translational initiation', 'RNA binding', 'cytoplasm', 'macromolecular complex', 'intracellular organelle part' and 'organelle part'. The KEGG (http://www.genome. $\mathrm{jp} / \mathrm{kegg} /$ ) pathways of up and downregulated genes are presented in Table III. The upregulated genes were primarily enriched in 'neuroactive ligand-receptor interaction', 
A

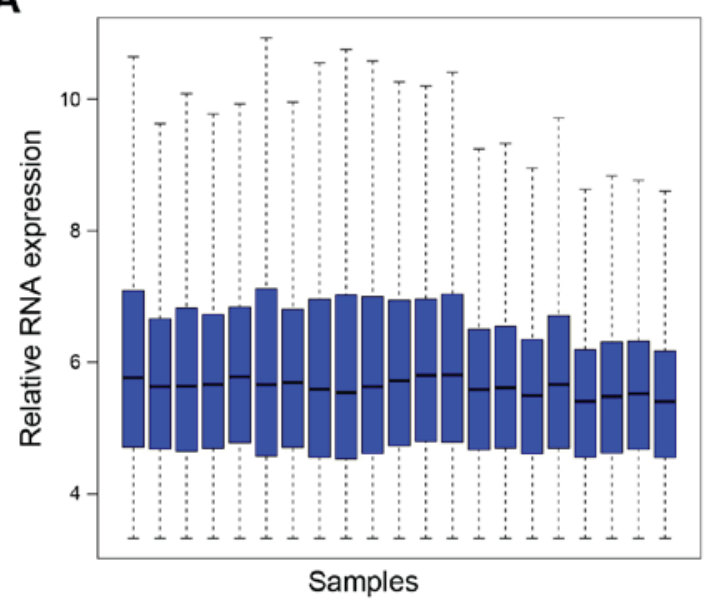

B

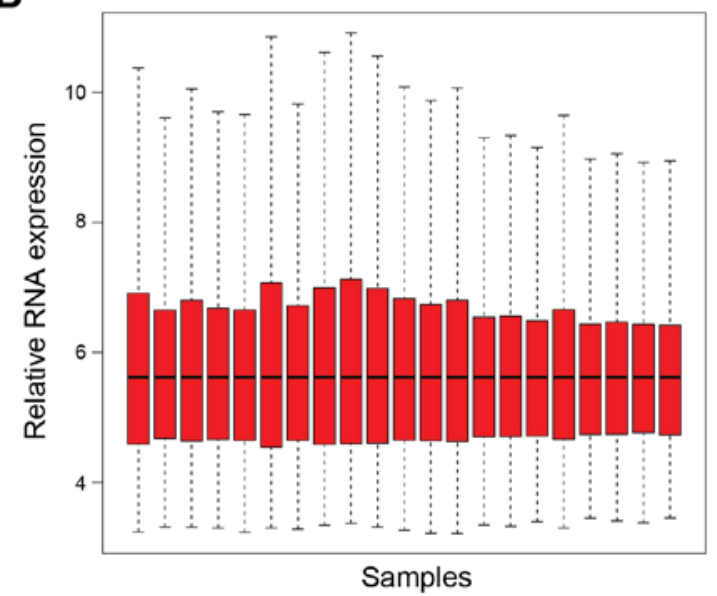

Figure 1. Box plots of data distribution in semen samples. The horizontal axis represents sample names and the vertical axis represents expression values. The first eight samples (left) are normal sperm samples. The reamining 13 samples are teratozoospermia samples. The black line in the box plot is the median of each data group and the data standardization degree may be inferred from its distribution. (A) Data prior to standardization. (B) Data following standardization.

A
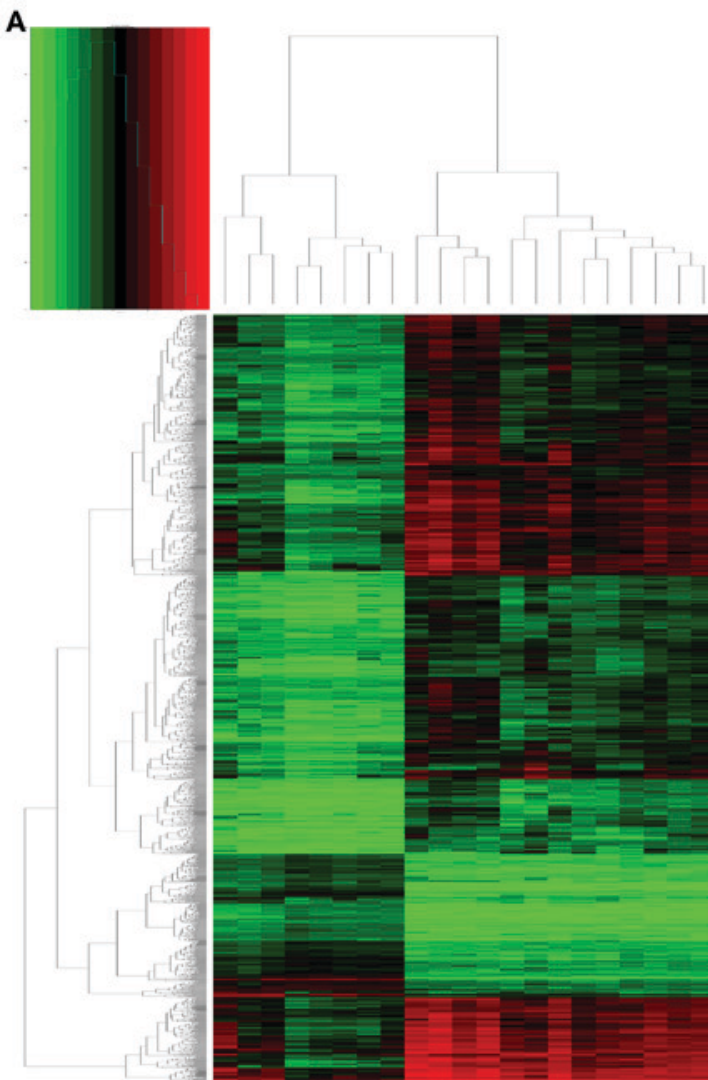

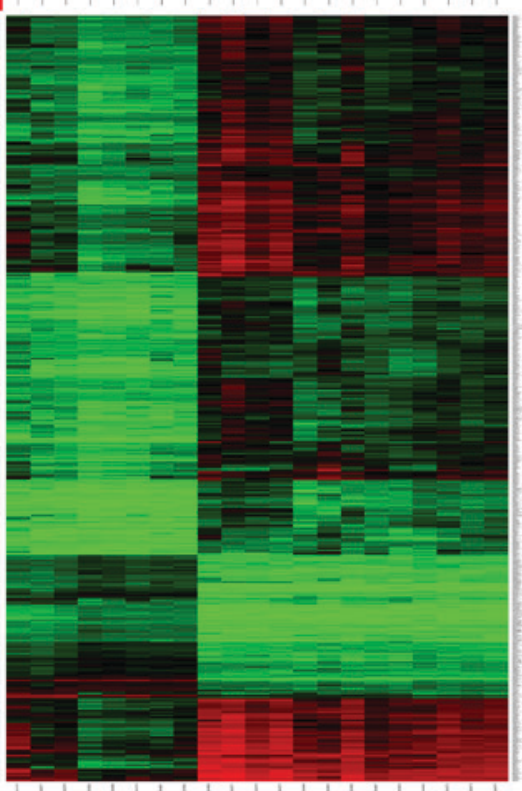

B

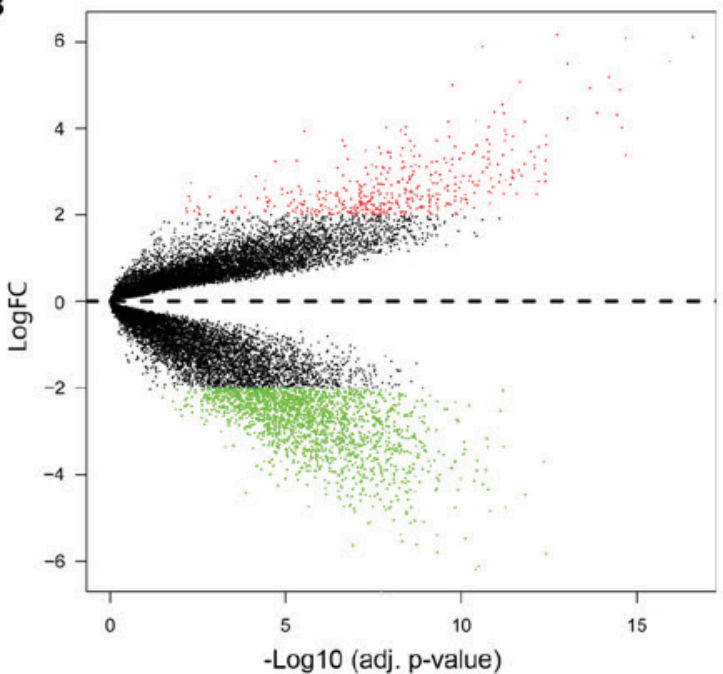

Figure 2. Differential expression analysis. (A) Heat map presenting the expression pattern across different samples. The horizontal axis represents sample names. The first eight samples are normal sperm samples. The further 13 samples are teratozoospermia samples. The left vertical axis presented clusters of DEGs, and the top horizontal axis presents clusters of samples. Red represents upregulated genes and green represents downregulated genes. (B) Volcano plot of DEGs. The $\mathrm{y}$-axis is $\operatorname{logFC}$ and the $\mathrm{x}$-axis represents - $\log 10$ (adjusted P-value). The red dots represent the DEGs upregulated and the green dots represent the DEGs downregulated while the black dots represent genes that were not differentially expressed. DEGs, differentially expressed genes; FC, fold change.

'retrograde endocannabinoid signaling', 'morphine addiction', 'GABAergic synapses', 'nicotine addiction', 'Rap1 signaling', 'Ras signaling', 'PI3K-Akt signaling', and 'glutamatergic and cholinergic synapses'. Down-regulated genes were associated with 'ribosomes', 'Huntington's disease', 'oxidative phosphorylation', 'Parkinson's and Alzheimer's diseases', 'proteasomes', 'non-alcoholic fatty liver disease', 'metabolic pathways', 'protein processing in the endoplasmic reticulum' and 'RNA transport'.

PPI network construction and module analysis. In order to extract PPI data, the present study uploaded 450 upregulated 
Table I. Top 10 upregulated and downregulated DEGs.

A, Upregulated DEGs

\begin{tabular}{lllclrr}
\hline Gene & logFC & AveExpr & $\mathrm{t}$ & P-value & Adjusted P-value & B \\
\hline HS3ST3A1 & 6.15829 & 6.264543 & 22.41724 & $1.07 \times 10^{-16}$ & $1.78 \times 10^{-13}$ & 28.22125 \\
XKR4 & 6.108402 & 6.904738 & 38.15492 & $1.13 \times 10^{-21}$ & $2.44 \times 10^{-17}$ & 38.46529 \\
C14orf37 & 6.089262 & 5.604961 & 29.23487 & $3.63 \times 10^{-19}$ & $1.97 \times 10^{-15}$ & 33.48849 \\
HYMAI & 5.891272 & 7.247713 & 16.23585 & $9.01 \times 10^{-14}$ & $2.38 \times 10^{-11}$ & 21.67427 \\
WIF1 & 5.55279 & 5.831665 & 34.46781 & $1.03 \times 10^{-20}$ & $1.11 \times 10^{-16}$ & 36.61418 \\
SRGAP2C & 5.491472 & 6.150009 & 23.34658 & $4.51 \times 10^{-17}$ & $8.89 \times 10^{-14}$ & 29.03932 \\
SRGAP2 & 5.182356 & 5.577265 & 26.86696 & $2.24 \times 10^{-18}$ & $6.06 \times 10^{-15}$ & 31.83706 \\
DQ592442 & 5.070846 & 7.77992 & 19.09086 & $3.15 \times 10^{-15}$ & $2.07 \times 10^{-12}$ & 24.96463 \\
MOGAT1 & 5.004399 & 5.407998 & 14.35237 & $1.10 \times 10^{-12}$ & $1.71 \times 10^{-10}$ & 19.18967 \\
LOC101928622 & 4.938621 & 5.265134 & 25.09824 & $9.64 \times 10^{-18}$ & $2.09 \times 10^{-14}$ & 30.48735
\end{tabular}

B, downregulated degs

\begin{tabular}{|c|c|c|c|c|c|c|}
\hline Gene & $\log \mathrm{FC}$ & AveExpr & $\mathrm{t}$ & P-value & Adjusted P-value & B \\
\hline ZPBP & -5.39989 & 9.272953 & -13.465 & $3.91 \times 10^{-12}$ & $4.71 \times 10^{-10}$ & 17.91695 \\
\hline PPDPF & -5.48137 & 7.809478 & -15.1896 & $3.50 \times 10^{-13}$ & $7.22 \times 10^{-11}$ & 20.32858 \\
\hline MSMB & -5.54533 & 8.057753 & -11.4953 & $8.49 \times 10^{-11}$ & $4.52 \times 10^{-09}$ & 14.82184 \\
\hline TSACC & -5.62232 & 9.854213 & -12.3248 & $2.22 \times 10^{-11}$ & $1.74 \times 10^{-09}$ & 16.17356 \\
\hline PIP & -5.63595 & 8.930515 & -9.11178 & $6.10 \times 10^{-09}$ & $1.17 \times 10^{-07}$ & 10.49482 \\
\hline TCEAL4 & -5.81103 & 7.351813 & -13.5001 & $3.72 \times 10^{-12}$ & $4.57 \times 10^{-10}$ & 17.96871 \\
\hline RPS5 & -5.83978 & 7.284755 & -21.5724 & $2.41 \times 10^{-16}$ & $3.71 \times 10^{-13}$ & 27.44499 \\
\hline RPL7AL2 & -6.12204 & 7.736477 & -16.0315 & $1.17 \times 10^{-13}$ & $3.01 \times 10^{-11}$ & 21.41787 \\
\hline SEMG1 & -6.1932 & 10.05513 & -15.8135 & $1.54 \times 10^{-13}$ & $3.67 \times 10^{-11}$ & 21.14096 \\
\hline SEMG2 & -6.69875 & 7.822177 & -10.4268 & $5.32 \times 10^{-10}$ & $1.81 \times 10^{-08}$ & 12.96753 \\
\hline
\end{tabular}

DEGs, differentially expressed genes; FC, fold change; HS3ST3A1, heparan sulfate-glucosamine 3-sulfotransferase 3A1; XKR4, XK related 4; C14orf37, armadillo-like helical domain containing 4; HYMAI, hydatidiform mole associated and imprinted (non-protein coding); WIF1, WNT inhibitory factor 1; SRGAP2C, SLIT-ROBO Rho GTPase activating protein 2C; SRGAP2, SLIT-ROBO Rho GTPase activating protein 2; MOGAT1, monoacylglycerol O-acyltransferase 1; ZPBP, zona pellucida binding protein; PPDPF, pancreatic progenitor cell differentiation and proliferation factor; MSMB, microseminoprotein $\beta$; TSACC, TSSK6 activating cochaperone; PIP, prolactin induced protein; TCEAL4, transcription elongation factor A like 4; RPS5, ribosome protein S5; RPL7AL2, ribosomal protein L7a pseudogene 12; SEMG1, semenogelin 1; SEMG2, semenogelin 2; AveExpr, Average expression of this probe set in all samples; $\mathrm{t}$, $\mathrm{t}$-value in the T-test between two sets of Bayes adjusted; $\mathrm{B}$, the logarithmic value of the standard deviation obtained by empirical Bayes.

genes and 1,942 downregulated genes to the STRING website. Subsequently, the samples with PPI data $>0.4$ were selected to assemble PPI networks. The PPI networks of upregulated genes are displayed in Fig. 3A. The upregulated network was constructed with 134 nodes and 199 edges. The $\mathrm{G}$ protein subunit $\beta 3$ (GNB3; degree=20), G protein subunit $\alpha$ ol (GNAO1; degree $=16$ ) and $\mathrm{G}$ protein subunit $\gamma$ transducin 1 (GNGT1; degree=15), were hub nodes in this network, which had almost twice the degree compared with other nodes in the network. The downregulated PPI network was subsequently constructed. The most significant modules were selected, with 160 nodes and 1,024 edges, as presented in Fig. 3B. Ribosomal protein S3 (RPS3; degree=32), RPS5 (degree=30), RPS16 (degree=29), RPS6 (degree=25) and RPS23 (degree=24) were hub nodes in this network.

\section{Discussion}

Over the last decade, the molecular mechanism underlying teratozoospermia has been of great research interest, with studies conducted in animal, human and cell models $(6,7)$. With the development of high-throughput technology, an increased number of genes/proteins have been demonstrated to be associated with male infertility $(23,24)$. However, a comprehensive understanding of how the biological processes at the molecular level are associated with the pathogenesis of teratozoospermia remains to be elucidated. Therefore, it is necessary to elucidate the latent pathogenesis of teratozoospermia at the systems biology level. The present study identified the disease module associated with teratozoospermia, systematically investigated the interaction 
Table II. Gene Ontology terms enriched in the teratozoospermia-related module.

A, Upregulated genes

\begin{tabular}{|c|c|c|c|}
\hline ID & Term & Count & FDR \\
\hline GO.0007399 & Nervous system development & 37 & $4.12 \times 10^{-07}$ \\
\hline GO.0032502 & Developmental process & 60 & $3.97 \times 10^{-07}$ \\
\hline GO.0048856 & Anatomical structure development & 56 & $3.52 \times 10^{-07}$ \\
\hline GO.0098794 & Postsynapse & 16 & $3.04 \times 10^{-07}$ \\
\hline GO.0050793 & Regulation of developmental process & 39 & $1.73 \times 10^{-07}$ \\
\hline GO.2000026 & Regulation of multicellular organismal development & 34 & $6.97 \times 10^{-08}$ \\
\hline GO.0097060 & Synaptic membrane & 15 & $6.89 \times 10^{-08}$ \\
\hline GO.0051094 & Positive regulation of developmental process & 30 & $1.85 \times 10^{-08}$ \\
\hline GO.0051239 & Regulation of multicellular organismal process & 44 & $1.85 \times 10^{-08}$ \\
\hline GO.0045211 & Postsynaptic membrane & 16 & $4.26 \times 10^{-10}$ \\
\hline
\end{tabular}

B, Downregulated genes

\begin{tabular}{|c|c|c|c|}
\hline ID & Term & Count & FDR \\
\hline GO.0045047 & Protein targeting to ER & 62 & $1.05 \times 10^{-37}$ \\
\hline GO.0031974 & Membrane-enclosed lumen & 497 & $3.44 \times 10^{-38}$ \\
\hline GO.0044428 & Nuclear part & 456 & $3.40 \times 10^{-38}$ \\
\hline GO.0006614 & SRP-dependent cotranslational protein targeting to membrane & 62 & $2.24 \times 10^{-38}$ \\
\hline GO.0006413 & Translational initiation & 89 & $6.39 \times 10^{-39}$ \\
\hline GO.0003723 & RNA binding & 262 & $8.87 \times 10^{-40}$ \\
\hline GO.0005737 & Cytoplasm & 941 & $2.62 \times 10^{-43}$ \\
\hline GO.0032991 & Macromolecular complex & 552 & $4.40 \times 10^{-48}$ \\
\hline GO.0044446 & Intracellular organelle part & 807 & $1.03 \times 10^{-54}$ \\
\hline GO.0044422 & Organelle part & 821 & $8.62 \times 10^{-55}$ \\
\hline
\end{tabular}

FDR, false discovery rate; ER, endoplasmic reticulum; SRP, signal recognition particle.

of module genes through pathway and network analyses and PPI data, and constructed a comprehensive and systematic framework to trace relevant genes.

There are several upregulated module genes that were observed to be involved in the pathogenesis of teratozoospermia, including GNB3, GNAO1 and GNGT1, all of them belong to the $G$ proteins family, also known as guanine nucleotide-binding proteins. It has been reported that $\mathrm{G}$ proteins are present in human spermatozoa, transmit various stimulation signals from outside the cell to its interior and are associated with propagation (25-27). The aforementioned studies indicate that $\mathrm{G}$ proteins serve a role in the maintenance of fertilization capacity in human and mouse sperm (28). The aforementioned three $G$ protein genes have not yet been associated with teratozoospermia; however, other members of the same class have been demonstrated to be necessary during spermatogenesis. Decreased expression of $G$ protein subunit $\alpha$ i2 $\left(\mathrm{G}_{\alpha \mathrm{i} 2}\right)$ was detected in low-motility spermatozoa with midpieces that were bent on themselves (29). Similarly, the activation of $\mathrm{G}_{\alpha \mathrm{i} 2}$ may affect the volume of ejaculated spermatozoa (11). Defective expression of GNA13 was observed in macrocephalic and global nucleus spermatozoa (30). The axonemal-associated localization within the midpiece and principal piece of various mammalian mature spermatozoa indicates that the G protein $\alpha$-subunit gustducin likely affects sperm motility via intracellular signal transduction (31).

A comparative study of epigenetic research between fertile and infertile boars indicated significantly increased DNA methylation levels in the GNAS complex locus of infertile boars (32). These data suggest that $G$ proteins may be downregulated in abnormal spermatogenesis. However, the results of the present study suggested that one of the $\mathrm{G}$ protein clusters that have never been proposed to have a function during spermatogenesis was enriched. GNB3, GNAO1 and GNGT1 are upregulated in sperm of patients with teratozoospermia, which may indicate a more comprehensive function of the $G$ protein during spermatogenesis.

In addition, various ribosomal genes, including RPS3, RPS5, RPS6, RPS16 and RPS23, were observed to be downregulated in abnormal sperm, in the present study. Prior to the present study, RPS3 had not been reported to be associated with spermatogenesis. A previous study suggested that RPS6 may regulate the viability of sertoli cells in blood-testis barrier 
Table III. KEGG pathways enriched in the teratozoospermia-related module.

A, Up-regulated genes

Term

Count

FDR

Neuroactive ligand-receptor interaction

$7.99 \times 10^{-08}$

Retrograde endocannabinoid signaling

$1.95 \times 10^{-06}$

Morphine addiction

$9.94 \times 10^{-06}$

GABAergic synapse

$8.70 \times 10^{-05}$

Nicotine addiction

$3.09 \times 10^{-04}$

Rap1 signaling pathway

$3.89 \times 10^{-04}$

Ras signaling pathway

$5.16 \times 10^{-04}$

PI3K-Akt signaling pathway

$5.16 \times 10^{-04}$

Glutamatergic synapse

$2.62 \times 10^{-03}$

Cholinergic synapse

$1.98 \times 10^{-02}$

\section{B, Down-regulated genes}

\begin{tabular}{lrr}
\hline Term & Count & FDR \\
\hline Ribosome & 63 & $1.12 \times 10^{-34}$ \\
Huntington s disease & 56 & $8.43 \times 10^{-19}$ \\
Oxidative phosphorylation & 46 & $3.97 \times 10^{-18}$ \\
Parkinson's disease & 45 & $4.14 \times 10^{-16}$ \\
Alzheimer's disease & 46 & $7.12 \times 10^{-14}$ \\
Proteasome & 20 & $1.94 \times 10^{-10}$ \\
Non-alcoholic fatty liver disease & 38 & $2.39 \times 10^{-10}$ \\
Metabolic pathways & 147 & $2.17 \times 10^{-09}$ \\
Protein processing in endoplasmic reticulum & 35 & $2.29 \times 10^{-07}$ \\
RNA transport & 30 & $1.65 \times 10^{-05}$ \\
\hline
\end{tabular}

KEGG, Kyoto Encyclopedia of Genes and Genomes; FDR, false discovery rate; GABA, $\gamma$-aminobutyric acid.

A

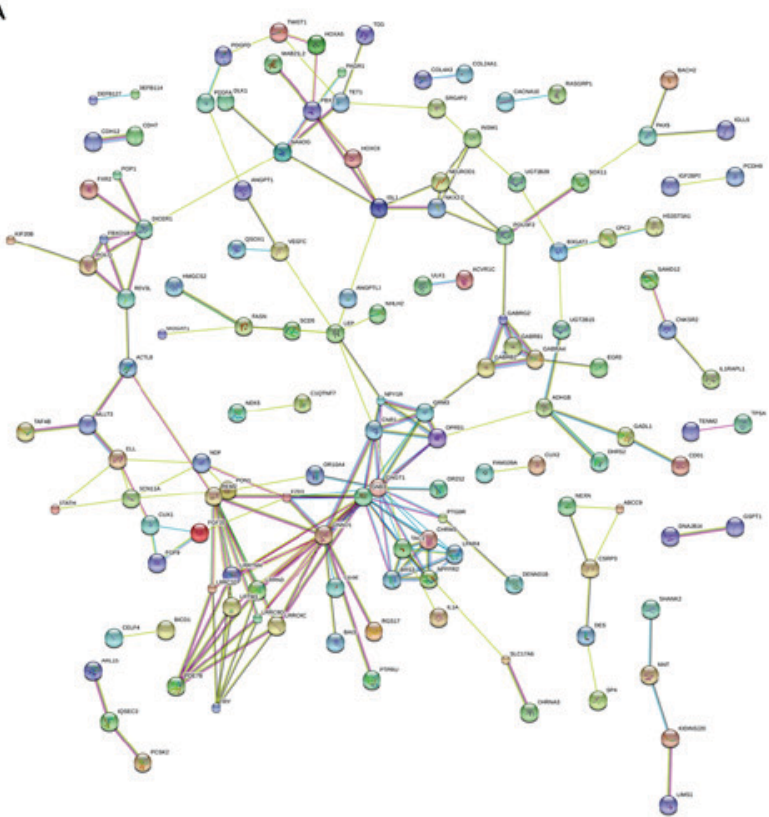

B

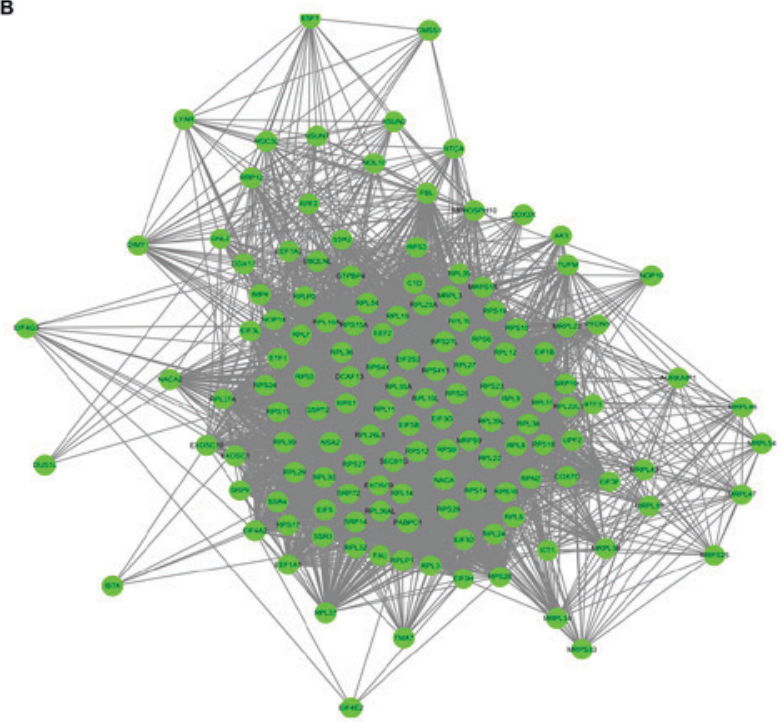

Figure 3. Network analysis of DEGs. (A) PPI network of upregulated DEGs obtained from the STRING database. Upregulated network was constructed with 134 nodes and 199 edges. (B) PPI network of downregulated DEGs obtained from the STRING database. The downregulated network was constructed with 160 nodes and 1,024 edges. DEGs, differentially expressed genes; PPI, protein-protein interaction. 
dynamics in rats (33). Furthermore, it has also been reported that RPS6 levels are downregulated via the serine/threonine-protein kinase mTOR signaling pathway in rats with sperm defects (34).

The function of the RPS23 gene, which is reported to be expressed in bovine sperm, remains to be fully elucidated (35). A previous study demonstrated that the downregulation of RPS16 and RPS5 in infertile patients is purportedly associated with asthenozoospermia (36). The consistency between previous studies and the results of the present study suggest that the methods used in the present study were effective in the study of teratozoospermia.

In conclusion, the present study used a systems biology framework for a comprehensive and systematic biological function- and network-based analysis of teratozoospermia. By integrating the information from GO, KEGG pathway and pathway crosstalk, it was revealed that three upregulated genes and five downregulated genes are enriched in the teratozoospermia-associated module. This systematic and comprehensive investigation of the teratozoospermia-associated module genes may improve the understanding of the contribution of genetic factors and their interactions with the pathogenesis of teratozoospermia, and may aid in identification of potential biomarkers for further investigation.

\section{Acknowledgements}

Not applicable.

\section{Funding}

The present study was supported by the Family Planning Project, Shanghai Municipal Commission of 415 Health and Family Planning (grant no. 201440002); Youth Foundation of Shanghai Institute of Planned Parenthood Research; Youth Foundation of Shanghai municipal commission of health and family planning (grant no. 20164Y0267).

\section{Availability of data and materials}

All data generated or analyzed during this study are included in this published article.

\section{Authors' contributions}

TZ and CL conducted data acquisition and analysis, and drafted the manuscript. JW and ZN contributed to the analysis of the results and revised the manuscript critically for important intellectual content. JZ and FY made substantial contributions to the design of the present study and critically revised the manuscript for important intellectual content.

\section{Ethics approval and consent to participate}

Not applicable.

\section{Consent for publication}

Not applicable.

\section{Competing interests}

The authors declare that they have no competing interests.

\section{References}

1. Browne RK, Kaurova SA, Uteshev VK, Shishova NV, McGinnity D, Figiel CR, Mansour N, Agney D, Wu M, Gakhova EN, et al: Sperm motility of externally fertilizing fish and amphibians. Theriogenology 83: 1-13, 2015.

2. Soulavie F, Piepenbrock D, Thomas J, Vieillard J, Duteyrat JL, Cortier E, Laurencon A, Gopfert MC and Durand B: Hemingway is required for sperm flagella assembly and ciliary motility in Drosophila. Mol Biol Cell 25: 1276-1286, 2014.

3. Sendler E, Johnson GD, Mao S, Goodrich RJ, Diamond MP, Hauser R and Krawetz SA: Stability, delivery and functions of human sperm RNAs at fertilization. Nucleic Acids Res 41: 4104-4117, 2013.

4. Georgiadis AP, Kishore A, Zorrilla M, Jaffe TM, Sanfilippo JS, Volk E, Rajkovic A and Yatsenko AN: High quality RNA in semen and sperm: isolation, analysis and potential application in clinical testing. J Urol 193: 352-359, 2015.

5. Ostermeier GC, Miller D, Huntriss JD, Diamond MP and Krawetz SA: Reproductive biology: Delivering spermatozoan RNA to the oocyte. Nature 429: 154, 2004.

6. Coutton C, Escoffier J, Martinez G, Arnoult C and Ray PF: Teratozoospermia: Spotlight on the main genetic actors in the human. Hum Reprod Update 21: 455-485, 2015.

7. De Braekeleer M, Nguyen MH, Morel F and Perrin A: Genetic aspects of monomorphic teratozoospermia: A review. J Assist Reprod Genet 32: 615-623, 2015.

8. Kang-Decker N, Mantchev GT, Juneja SC, McNiven MA and van Deursen JM: Lack of acrosome formation in Hrb-deficient mice. Science 294: 1531-1533, 2001.

9. Xu X, Toselli PA, Russell LD and Seldin DC: Globozoospermia in mice lacking the casein kinase II alpha' catalytic subunit. Nat Genet 23: 118-121, 1999.

10. Sadek CM, Damdimopoulos AE, Pelto-Huikko M, Gustafsson JA, Spyrou G and Miranda-Vizuete A: Sptrx-2, a fusion protein composed of one thioredoxin and three tandemly repeated NDP-kinase domains is expressed in human testis germ cells. Genes Cells 6: 1077-1090, 2001.

11. Allegrucci C, Liguori L and Minelli A: Stimulation by n6-cyclopentyladenosine of $\mathrm{A} 1$ adenosine receptors, coupled to galphai2 protein subunit, has a capacitative effect on human spermatozoa. Biol Reprod 64: 1653-1659, 2001.

12. Matzuk MM and Lamb DJ: Genetic dissection of mammalian fertility pathways. Nat Cell Biol 4 (Suppl): S41-S49, 2002.

13. O'Doherty AM and McGettigan PA: Epigenetic processes in the male germline. Reprod Fertil Dev 27: 725-738, 2015.

14. H MY, Kumar S, Dubey PP, Modi RP, Chaudhary R, A SK, Ghosh SK, Sarkar M and B S: Profiling of sperm gene transcripts in crossbred (Bos taurus x Bos indicus) bulls. Anim Reprod Sci 177: 25-34, 2017.

15. Hu K, Zhang J and Liang M: LncRNA AK015322 promotes proliferation of spermatogonial stem cell C18-4 by acting as a decoy for microRNA-19b-3p. In Vitro Cell Dev Biol Anim 53: 277-284, 2017.

16. Kherraf ZE, Christou-Kent M, Karaouzene T, Amiri-Yekta A, Martinez G, Vargas AS, Lambert E, Borel C, Dorphin B, Aknin-Seifer I, et al: SPINK2 deficiency causes infertility by inducing sperm defects in heterozygotes and azoospermia in homozygotes. EMBO Mol Med 9: 1132-1149, 2017.

17. Platts AE, Dix DJ, Chemes HE, Thompson KE, Goodrich R, Rockett JC, Rawe VY, Quintana S, Diamond MP, Strader LF and Krawetz SA: Success and failure in human spermatogenesis as revealed by teratozoospermic RNAs. Hum Mol Genet 16: 763-773, 2007.

18. Huang da W, Sherman BT and Lempicki RA: Systematic and integrative analysis of large gene lists using DAVID bioinformatics resources. Nat Protoc 4: 44-57, 2009.

19. Ashburner M, Ball CA, Blake JA, Botstein D, Butler H, Cherry JM, Davis AP, Dolinski K, Dwight SS, Eppig JT, et al: Gene ontology: Tool for the unification of biology. The gene ontology consortium. Nat Genet 25: 25-29, 2000.

20. Sanchez MC, Sedo CA, Julianelli VL, Romanato M, Calvo L, Calvo JC and Fontana VA: Dermatan sulfate synergizes with heparin in murine sperm chromatin decondensation. Syst Biol Reprod Med 59: 82-90, 2013. 
21. Langsdorf A, Schumacher V, Shi X, Tran T, Zaia J, Jain S, Taglienti M, Kreidberg JA, Fine A and Ai X: Expression regulation and function of heparan sulfate 6-O-endosulfatases in the spermatogonial stem cell niche. Glycobiology 21: 152-161, 2011.

22. Lin YN, Roy A, Yan W, Burns KH and Matzuk MM: Loss of zona pellucida binding proteins in the acrosomal matrix disrupts acrosome biogenesis and sperm morphogenesis. Mol Cell Biol 27: 6794-6805, 2007.

23. Zhu Y, Ma M, Wan L, Zhang D, Zhao L, Wei L and Li L: Analysis of DAZL SNP260 and SNP386 in infertile Chinese males using multi-analyte suspension array. Mol Med Rep 10: 2949-2954, 2014.

24. Li YZ, Chen ZY, Wang H, Huang H, Song QX and Zhou GH: Establishment of a hydrogel chip for high-throughput detection of Y chromosome microdeletions. Zhonghua Nan Ke Xue 18: 109-114, 2012 (In Chinese).

25. Hinsch KD, Schwerdel C, Habermann B, Schill WB, Muller-Schlosser F and Hinsch E: Identification of heterotrimeric $\mathrm{G}$ proteins in human sperm tail membranes. Mol Reprod Dev 40: 345-354, 1995.

26. Merlet F, Weinstein LS, Goldsmith PK, Rarick T, Hall JL, Bisson JP and de Mazancourt P: Identification and localization of G protein subunits in human spermatozoa. Mol Hum Reprod 5: $38-45,1999$.

27. Modarressi MH, Taylor KE and Wolfe J: Cloning, characterization, and mapping of the gene encoding the human $\mathrm{G}$ protein gamma 2 subunit. Biochem Biophys Res Commun 272: 610-615, 2000.

28. Baxendale RW and Fraser LR: Immunolocalization of multiple galpha subunits in mammalian spermatozoa and additional evidence for galphas. Mol Reprod Dev 65: 104-113, 2003.

29. Hu Y, Lu Y, Zhou Z, Du Y, Xing J, Wang L, Lin M and Sha J: Defective expression of Galpha12 in the testes of azoospermia patients and in the spermatozoa with low motility. J Mol Med (Berl) 84: 416-424, 2006.
30. Hu Y, Xing J, Chen L, Guo X, Du Y, Zhao C, Zhu Y, Lin M, Zhou Z and Sha J: RGS22, a novel testis-specific regulator of G-protein signaling involved in human and mouse spermiogenesis along with GNA12/13 subunits. Biol Reprod 79: 1021-1029, 2008.

31. Fehr J, Meyer D, Widmayer P, Borth HC, Ackermann F, Wilhelm B, Gudermann T and Boekhoff I: Expression of the G-protein alpha-subunit gustducin in mammalian spermatozoa. J Comp Phys A Neuroethol Sens Neural Behav Physiol 193: 21-34, 2007.

32. Congras A, Yerle-Bouissou M, Pinton A, Vignoles F, Liaubet L, Ferchaud S and Acloque H: Sperm DNA methylation analysis in swine reveals conserved and species-specific methylation patterns and highlights an altered methylation at the GNAS locus in infertile boars. Biol Reprod 91: 137, 2014.

33. Mok KW, Chen H, Lee WM and Cheng CY: rpS6 regulates blood-testis barrier dynamics through Arp3-mediated actin microfilament organization in rat sertoli cells. An in vitro study. Endocrinology 156: 1900-1913, 2015.

34. Xu H, Shen L, Chen X, Ding Y, He J, Zhu J, Wang Y and Liu X mTOR/P70S6K promotes spermatogonia proliferation and spermatogenesis in Sprague Dawley rats. Reprod Biomed Online 32: 207-217, 2016.

35. Han CM, Chen R, Li T, Chen XL, Zheng YF, Ma MT and Gao QH: Evaluation of the semen swim-up method for bovine sperm RNA extraction. Genet Mol Res 15: gmr.15027713, 2016.

36. Bansal SK, Gupta N, Sankhwar SN and Rajender S: Differential genes expression between fertile and infertile spermatozoa revealed by transcriptome analysis. PloS One 10: e0127007, 2015.

This work is licensed under a Creative Commons Attribution-NonCommercial-NoDerivatives 4.0 International (CC BY-NC-ND 4.0) License. 